\title{
Development of a Mobile Modular System for the Detection of Special Nuclear Material (MODES_SNM)
}

\author{
D. Cester, M. Lunardon, L. Stevanato, G. Viesti ${ }^{1}$
}

Dipartimento di Fisica ed Astronomia dell' Università di Padova Via Marzolo 8, 35131 Padova, Italy

R. Chandra, G. Davatz, H. Friederich, U. Gendotti, D. Murer

Arktis Radiation Detectors Ltd., Switzerland

\section{Swiderski, M. Moszynski}

National Centre for Nuclear Research, Soltana 7, 05-400 Otwock-Swierk, Poland

\section{F. Resnati, A. Rubbia}

ETH Zurich, Institute for Particle Physics, Zurich, Switzerland

\author{
A. lovene, S. Petrucci, C. Tintori \\ CAEN S.p.A., Via Vetraia 11, I-55049, Viareggio, LU, Italy
}

M. Caccia, V. Chmill, R. Santoro, A. Martemyianov ${ }^{2}$

Dipartimento di Scienza e Alta Tecnologia, Universita dell'Insubria, Como, Italy

\section{Doherty}

Revenue Commissioners Ireland, Ashtowngate, Navan Road, Dublin 15, Ireland.

\section{G. Christodoulou, T. Stainer, C. Touramanis}

University of Liverpool, Liverpool L69 7ZE, United Kingdom

The project MODES_SNM, Modular DEtection System for Special Nuclear Material, is presented in this paper. Its main objective is the development of a mobile and modular detection system specially designed for SNM, i.e. highly enriched Uranium and weapon grade Plutonium, but with the capability of detecting and identifying radioactive sources. The MODES_SNM prototype is defined as a Portable Radiation Scanner following the IAEA classification that can be used in primary or secondary lines of control depending on the operational need as fixed or mobile system. High pressure noble gas scintillation detectors for neutrons and gamma rays are employed in the detection system.

X Latin American Symposium on Nuclear Physics and Applications (X LASNPA)

1-6 December 2013

Montevideo, Uruguay

\footnotetext{
${ }^{1}$ Speaker E-mail: giuseppe.viesti@unipd.it

${ }^{2}$ Permanent address: ITEP, Bolshaya Cheremushkinskaya, 25, 117218 Moscow Russia
} 


\section{Introduction}

MOdular DEtection System for Special Nuclear Materials (MODES-SNM) [1] is a FP7 European funded project aiming at the design, the construction and the laboratory qualification of a mobile system for the detection of radioactive sources and Special Nuclear Materials (SNM). The device was designed to fulfill the IAEA standard of a Portable Radiation Scanner (PRS) [2], which is defined as a device designed mainly for covert detection of radioactive materials in situations when the use of standard radiation portals is not possible. The PRS shall be used in stationary as well as mobile modes and is capable of detecting an anomalous increase of the background radiation and identifying the source of the extra yield. The MODES_SNM project will be completed in mid 2014 after a field test campaign in different EU seaports.

The MODES_SNM Consortium includes four Universities (Università degli Studi di Padova (Italy), Eidgenoessische Technische Hochschule Zurich (Switzerland), Università degli Studi dell' Insubria (Italy) and The University of Liverpool (United Kingdom)), two high technology companies Arktis Radiation Detectors LTD (Switzerland) and Costruzioni Apparecchiature Elettroniche Nucleari C.A.E.N. SpA (Italy) and the National Centre for Nuclear Research (Poland). Finally the Revenue Commissioners (Ireland) represents the enduser point of view inside the MODES_SNM Consortium. The University of Padova is the Project Coordinator.

\section{The MODES_SNM prototype}

The MODES_SNM prototype consists of an array of gamma ray and neutron (thermal and fast) detectors. The detection of fast neutrons is a peculiar choice of our system with respect to the well known ${ }^{3} \mathrm{He}$ proportional counters embedded in a polyethylene moderator, mainly used in radiation portals. It is motivated by the reduction of the natural neutron background in the energy range of fast neutrons (i.e. for a fission neutron spectrum) with respect to the typical systems based on ${ }^{3} \mathrm{He}$ proportional counters with polyethylene moderators which have a very large energy response and thus are more sensitive to the natural neutron background. Systems with an extended response at low energy are characterized by a larger background causing the decrease of the signal-to-noise ratio. The measure of the fast component of the neutron spectrum provides also additional information since it might allow, in principle, to discriminate fission neutron sources from (alpha,n) ones (as the $\mathrm{Am} / \mathrm{Be}$ or the $\mathrm{Pu} / \mathrm{Be}$ sources). Once the primary neutron inspection is performed in the MODES_SNM prototype by the fast neutron detectors, additional information is obtained from the thermal neutron detectors that work without moderator so that the measured fast-to-thermal neutron ratio brings information on the presence of shielding around the neutron source. The capability of providing information on the type of neutron source and on its eventual shielding is a peculiar characteristic of the MODES_SNM system. 


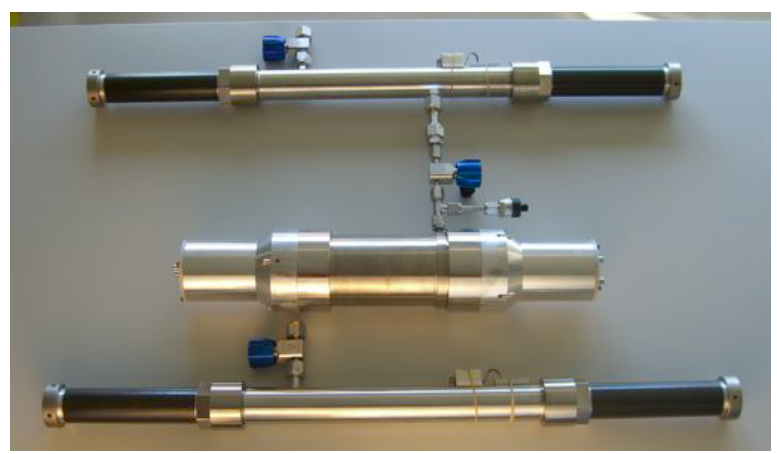

Fig.1 MODES_SNM detectors: fast neutron (top), gamma ray (middle) and thermal neutron(bottom) .

The common technology for the MODES-SNM detector suite is represented by high pressure cells filled with noble gases (xenon and 4-helium) used as scintillators. The 4-helium detectors for fast neutrons is pre-existing know-how by Arktis [3], but a new mechanical design of such detectors together with the development of the xenon detector for gamma-rays and a new helium detector for thermal neutrons represent relevant results of the MODES_SNM project. The detectors are shown in Fig.1. In parallel with the development of the detectors, a new front-end based on compact, low power digital electronics has been developed by CAEN [4] including desktop high voltage (HV) power supplies and new desktop $500 \mathrm{MSample/s,} 14$ bit resolution fast digitizers. The sampling rate was selected to optimize the neutron/gamma pulse shape discrimination based on the integration method [5].

A typical pulse shape discrimination (PSD) plot for the 4-Helium fast neutron detector is shown in Fig.2. The gamma ray sensitivity of this detector is lower with respect to liquid scintillators so that neutrons are more easily detected also in presence of a very strong gamma ray background, as required in case of Homeland Security applications.

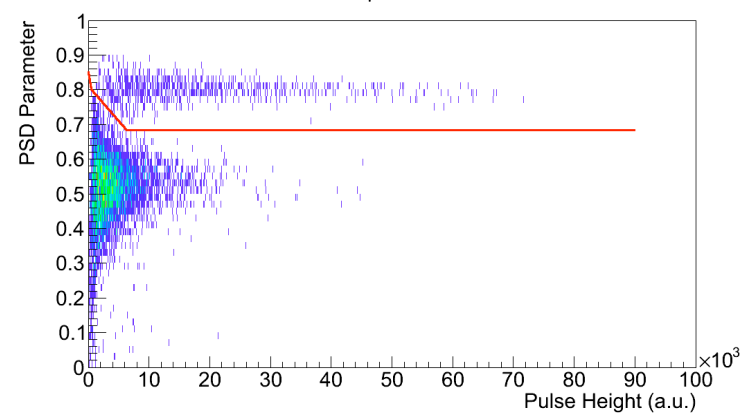

Fig.2 Typical PSD plot from the 4-He detectors showing the discrimination between neutrons (above the red line) and gamma rays (below the red line) from a ${ }^{252} \mathrm{Cf}$ source.

A special version of the 4-Helium detectors has been specifically developed including a $\mathrm{Li}$ converter to detect also slow neutrons that are discriminated from the fast component by a novel technique illustrated in Fig.3. 


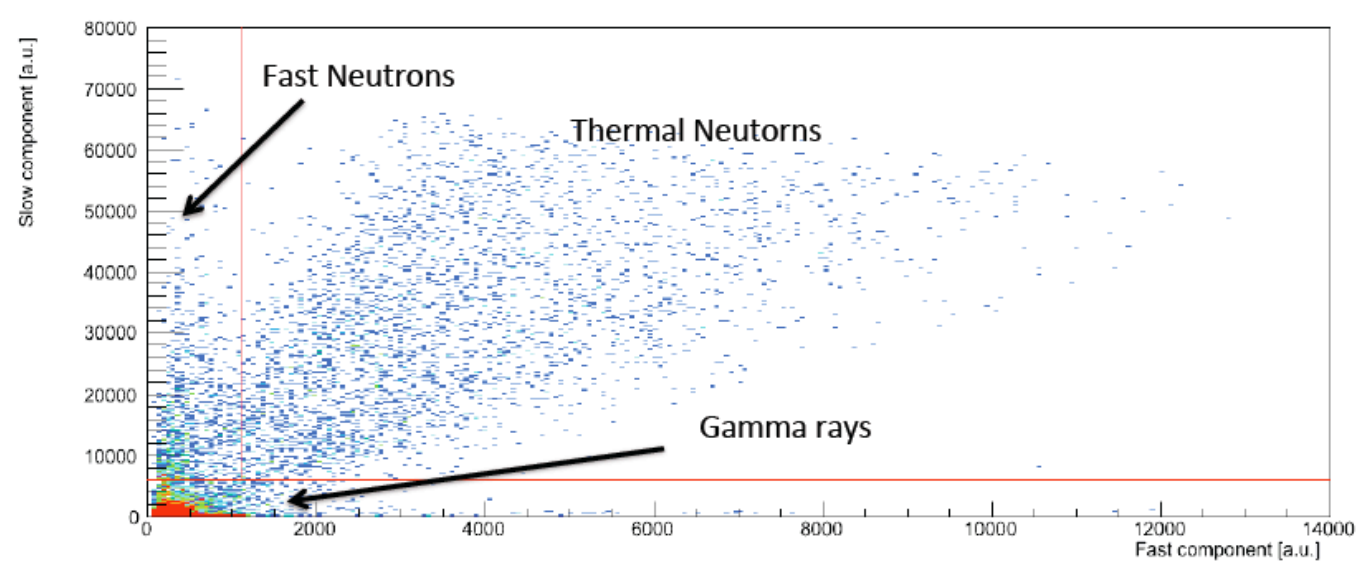

Fig.3 Discrimination of fast and thermal neutrons in the thermal neutron detector.

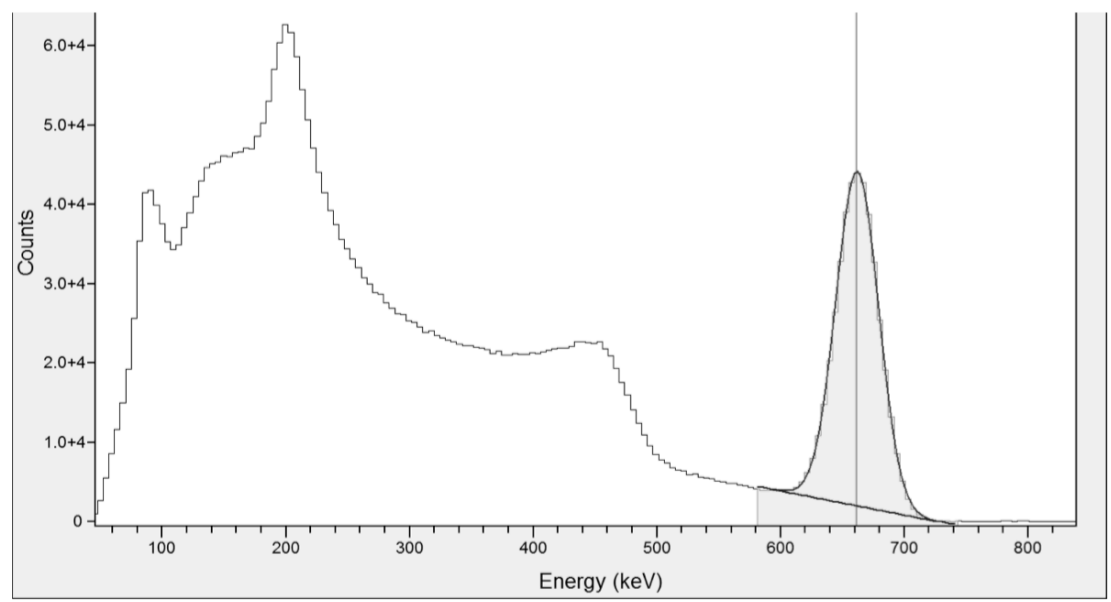

Fig.4 Typical gamma ray spectrum from a ${ }^{137}$ Cs source measured with the MODES_SNM $\mathrm{Xe}$ scintillation detector. The energy resolution is about $6 \%$ at $662 \mathrm{keV}$.

Xenon is an attractive material for gamma ray detection, mainly because of its high atomic number $(Z=54)$ and large cross-section for photo-electric absorption (photo-electric crosssection dominates up to around $300 \mathrm{keV}$ ) [6]. Looking to the scintillation light, the xenon detector is not susceptible to vibrational disturbance and micro-phonic effects typically associated to previously developed detectors using Ionization Chamber technology. The MODES_SNM gamma ray system is capable of reaching energy resolutions typical of the standard $\mathrm{NaI}(\mathrm{Tl})$ scintillators (see Fig. 4) but with larger volumes and lower costs so that it can be adapted easily to specific geometrical requirements. However, the peak efficiency of the Xe detector seems to be too low for gamma-rays around $1 \mathrm{MeV}$ so that in the final MODES_SNM system will use also a standard $\mathrm{NaI}(\mathrm{Tl})$ detector, complementing the Xe-based system.

It is also worth mentioning that the current MODES_SNM detectors make use of standard PMTs but a new read-out employing Silicon Photomultipliers (SiPM) has been tested 
successfully [7]. The future use of SiPM will improve costs, ruggedness and lay-out of the detection systems using high pressure scintillation cells.

All sub-components of the MODES_SNM system have been developed and integrated. The final prototype is currently being tested in laboratory conditions and will be soon assembled in a van-mounted configuration. The full system during the laboratory tests is shown in Fig. 5. The lower box contains the front-end electronics and the computer for the slow control, the data acquisition and analysis and the communication module for remote control. Starting to the left, the first five boxes contain neutron detectors and the last two boxes are the ones for gamma rays.

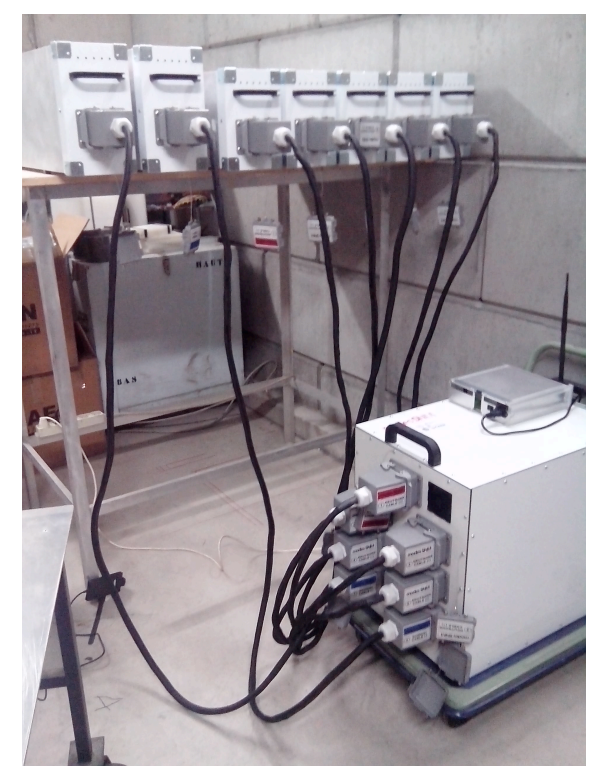

Fig.5 The MODES_SNM system during laboratory tests.

As an example of the system capability, we show in Fig.6 the frequency distribution of neutron counts for the natural background as well as the one for an irradiation with a ${ }^{252} \mathrm{Cf}$ source delivering 0.1 neutron/ $\left(\mathrm{s} \times \mathrm{cm}^{2}\right)$ on the detector front face. Integration time is $2 \mathrm{~s}$. The two distributions are well separated so that it is possible to set an alarm threshold for a detection probability larger than $99 \%$. The same representation is shown in Fig. 7 for a gamma ray measurement by using a ${ }^{133} \mathrm{Ba}$ source delivering a dose rate of $50 \mathrm{nSv} / \mathrm{h}$ on the front face of the $\mathrm{Xe}$ detector box. In this case the left panel shows the frequency distribution for the natural gamma ray background (blue) and the one corresponding to the ${ }^{133} \mathrm{Ba}$ source (red). Also in this case the integration time is $2 \mathrm{~s}$. The two frequency distributions are very well separated so that also in this case the detection capability larger than $99 \%$ should be achieved. Once the gamma ray alarm is detected, the system shall perform a second acquisition of the data, $60 \mathrm{~s}$ long. The derived gamma ray spectrum is analyzed by a specific software that performs the following actions: subtraction of the measured natural background; subtraction of the continuous part of the spectrum, mainly from the Compton events; identification and fit of the gamma ray peaks; selection of the transitions and identification of the emitters. An example of this procedure is shown in the left panel of Fig. 7 for the ${ }^{133} \mathrm{Ba}$ source at the dose rate of $50 \mathrm{nSv} / \mathrm{h}$. 


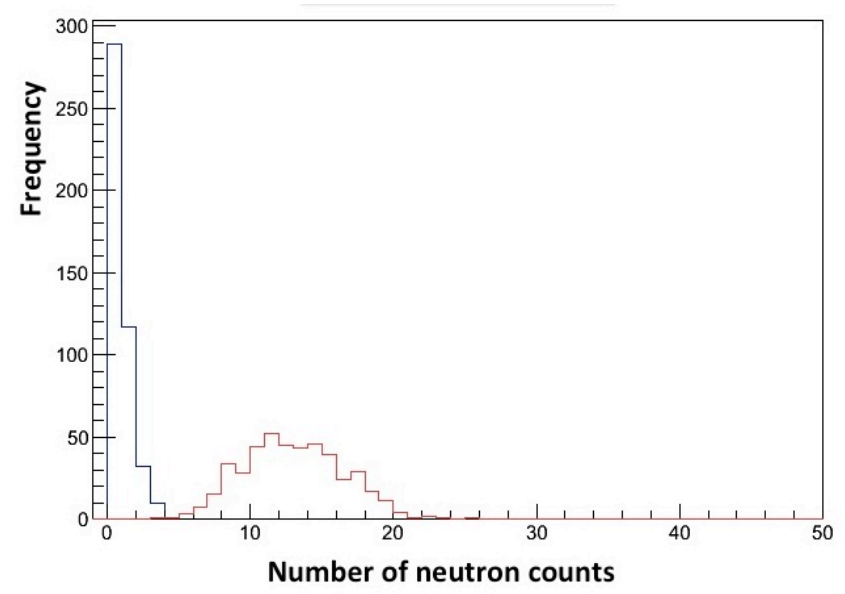

Fig.6 Frequency distribution of neutron counts for $2 \mathrm{~s}$ sampling windows for the natural background (blue line) and ${ }^{252} \mathrm{Cf}$ source (red line). For details see the text.
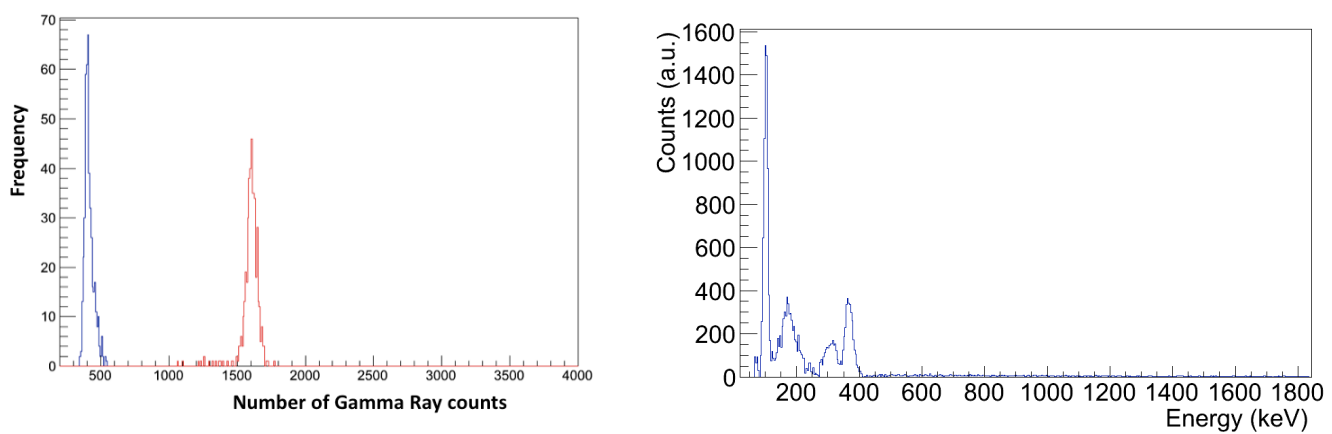

Fig.7. As Fig.6 but for gamma rays from a ${ }^{133} \mathrm{Ba}$ source in a Xe detector (left panel). Identification spectra for the ${ }^{133} \mathrm{Ba}$ source (right panel). For details see the text.

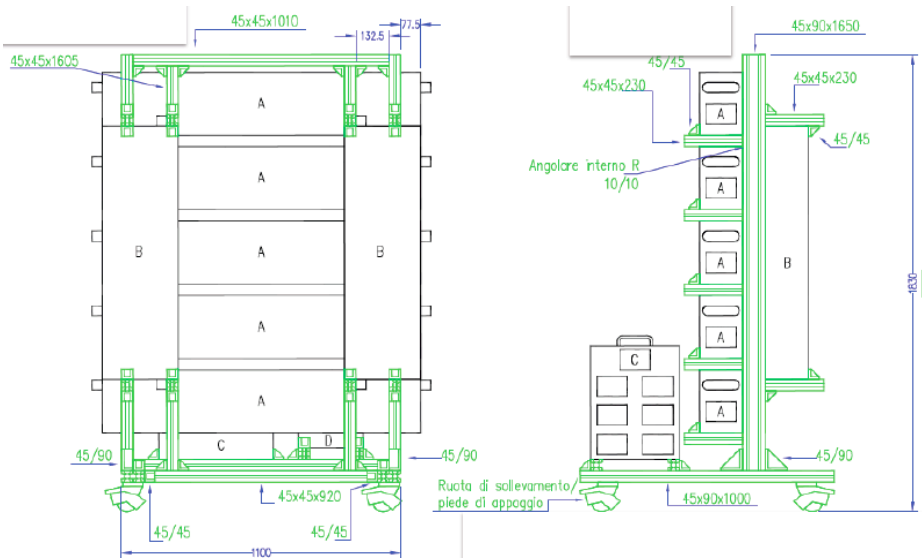

Fig.8 Lay-out of the MODES_SNM detector array. The vertical boxes contain the gamma ray detectors. 


\section{Conclusions}

The MODES_SNM prototype is in an advanced stage of preparation. The detector box will be soon assembled using a mechanical structure presented in Fig. 8 that will allow the use of the prototype as a van mounted system as well as mobile device that can be transported and operated in several scenarios. It can be operated using batteries as well as normal power line and controlled remotely by using any device with a web browser. Complete characterization of the prototype is planned in April 2014 at the JRC Ispra to verify the compliance with international standards.

\section{Acknowledgements}

This project has received funding from the European Union's Seventh Framework Programme for research, technological development and demonstration under Grant Agreement No 284842.

\section{References}

[1] See the web site www.modes-snm.eu

[2] IAEA Nuclear Security Series No. 1: Technical and functional specifications for border monitoring equipment. See http://www-ns.iaea.org/security/nss-publications.asp?s=5

[3] R.Chandra et al, 2012 Journal of Instrumentation 7 C03035 doi:10.1088/17480221/7/03/C03035; further documentation available at www.arktis-detectors.com

[4] documentation available at www.caen.it

[5] D. Cester et al., Nuclear Instruments and Methods A 748 (2014) 33-38.

[6] F. Resnati et al., Nuclear Instruments and Methods A715 (2013) 87-91.

[7] M. Caccia et al., 3rd International Conference on Advancements in Nuclear Instrumentation Measurement Methods and their Applications (ANIMMA), 2013 , DOI: http://dx.doi.org/10.1109/ANIMMA.2013.6727974 